\title{
Reduced intestinal FADS1 gene expression and plasma omega-3 fatty acids following Roux-En-Y Gastric Bypass
}

Authors: Priscila Garla ${ }^{1}$, Priscila Sala ${ }^{1}$, Raquel Susana Matos de Miranda Torrinhas $^{1}$, Natasha Mendonça Machado ${ }^{1}$, Danielle Cristina Fonseca ${ }^{1}$, Mariane Marques da Silva ${ }^{1}$, Graziela Rosa Ravacci ${ }^{1}$, Giliane Belarmino, Robson Kiyoshi Ishida $^{1,2}$, Ismael Francisco Mota Siqueira Guarda ${ }^{1}$, Eduardo Guimarães Hourneaux de Moura ${ }^{1,2}$, Paulo Sakai ${ }^{1,2}$, Marco Aurélio Santo ${ }^{1,2}$, Ismael Dale Cotrim Guerreiro da Silva ${ }^{3}$, Claudia Cristina Alves Pereira ${ }^{3}$, Steven Heymsfield ${ }^{4}$, Maria Lúcia Cardillo Corrêa-Giannella5,6, Philip C. Calder ${ }^{7}$, Dan Linetzky Waitzberg ${ }^{1,2}$

Author affiliations: ${ }^{1}$ Departamento de Gastroenterologia, Laboratório Metanutri (LIM 35), Faculdade de Medicina FMUSP, Universidade de Sao Paulo, Sao Paulo, SP, BR; ${ }^{2}$ Hospital das Clinicas HCFMUSP, Faculdade de Medicina, Universidade de São Paulo, Sao Paulo, SP, BR; ${ }^{3}$ Federal University of São Paulo, São Paulo, SP, BR; ${ }^{4}$ Pennington Biomedical Research Center, Baton Rouge, Louisiana, USA; ${ }^{5}$ Programa de Pós-Graduação em Medicina, Universidade Nove de Julho, Brazil; ${ }^{6}$ Laboratório de Carboidrato e Radioimunoensaio (LIM-18), Faculdade de Medicina FMUSP, Universidade de Sao Paulo, Sao Paulo, SP, BR, ${ }^{7}$ Faculty of Medicine, University of Southampton and National Institute for Health Research Southampton Biomedical Research Centre, University Hospital Southampton NHS Foundation Trust and University of Southampton, Southampton, United Kingdom. 
Authors' last names: Garla P, Sala P, Torrinhas RS, Machado NM, Fonseca DC, da Silva MM, Ravacci GR, Belarmino G, Ishida RK, Guarda IF, de Moura EG, Sakai P, Santo MA, da Silva ID, Rodrigues AS, Pereira CA, Heymsfield S, Correa-Giannella ML, Calder PC, Waitzberg DL

Corresponding Author: Priscila Garla

Av. Dr. Arnaldo, 455, $2^{\circ}$ andar, sala 2208 - Cerqueira César

Post code: 01246-903, São Paulo - SP, Brazil;

Phone / Fax: +55 11 3061-7459. E-mail: prigarla@gmail.com

Source of Support: This study was supported by Fapesp - Fundação de Amparo a Pesquisa do Estado de São Paulo (process number 2011/09612-3)

Running Title: Low omega-3 fatty acids after gastric bypass

Clinical Trial Registry number and website:

www.clinicaltrials.gov -NCT01251016;

Plataforma Brasil -19339913.0.0000.0068 
1 ABSTRACT: Roux-en-Y gastric bypass (RYGB) limits food ingestion and may

2 alter the intestinal expression of genes involved in the endogenous synthesis of

3 polyunsaturated fatty acids (PUFAs). These changes may decrease the systemic availability of bioactive PUFAs after RYGB. AIM: To study the impact of RYGB on the dietary ingestion and plasma concentration of PUFAs and on the intestinal expression of genes involved in their endogenous biosynthesis in severely obese women with type 2 diabetes. METHODS: Before, and 3 and 12 months after RYGB, obese women ( $n=20)$ self-reported a seven-day dietary record, answered a food frequency query and provided plasma samples for alpha-linolenic (ALA), eicosapentaenoic (EPA), docosahexaenoic (DHA) and arachidonic (ARA) acid assessment by gas chromatography. Intestinal biopsies (duodenum, jejunum and ileum) were collected through double-balloon endoscopy before and 3 months after RYGB for gene expression analysis by microarray (Human GeneChip 1.0 ST array) and RT-qPCR validation. RESULTS: Compared to the preoperative period, patients had decreased intakes of PUFAs, fish and soybean oil $(p<0.05)$ and lower plasma concentrations of ALA and EPA $(p<0.001) 3$ and 12 months after RYGB. FADS1 gene expression was lower in duodenum (RT-qPCR fold change $=-1.620, p<0.05)$ and jejunum $(R T-q P C R$ fold change $=-1.549, p<0.05) 3$ months following RYGB, compared to before surgery. CONCLUSION: RYGB decreased PUFA ingestion, plasma ALA and EPA levels, and intestinal expression of FADS1 gene. The latter encodes a key enzyme involved in endogenous biosynthesis of PUFAs. These data suggest that supplementation of omega-3 PUFAs may be required for obese patients undergoing RYGB.

Keywords: FADS1, omega-3 fatty acids, obesity, Roux-en-Y gastric bypass. 


\section{INTRODUCTION}

It is widely recognized that omega-3 (n-3) polyunsaturated fatty acids (PUFAs), in particular eicosapentaenoic acid (EPA; 20:5n-3) and docosahexaenoic acid (DHA; 22:6n-3), play an important protective role in metabolic, cardiovascular, developmental, and cognitive health. ${ }^{[1-3]}$ EPA and DHA are endogenously produced from the essential alpha-linolenic acid (ALA; 18:3n3) in a well-characterized enzymatic pathway (Figure 1). ${ }^{[4]}$ The conversion occurs by a sequential insertion of new carbons and double-bonds within the ALA chain, for which the enzymes encoded by the genes elongase 2 and 5 (ELOVL2 and ELOVL5, respectively) and fatty acid desaturase 1 and 2 (FADS1 and FADS2, respectively) play a crucial role. ${ }^{[4,5]}$ The same pathway is responsible for producing arachidonic acid (ARA; 20:4n-6) from its essential precursor linoleic acid (LA; 18:2n-6). This pathway is considered to mainly operate in the liver. However, human intestinal cell lines have been shown to desaturate and elongate LA and ALA to produce longer chain, more unsaturated derivatives. ${ }^{[6]}$ Although evidence of the contribution of the intestine to production of the bioactive $n-6$ and $\mathrm{n}-3$ PUFAs in humans is lacking, we suggest that the intestine may be an important secondary site for such synthesis.

Clinical trials and meta-analyses have demonstrated that genetic variation in the FADS1 and FADS2 genes influences the endogenous conversion of ALA to its bioactive derivatives. ${ }^{[5,7-9]}$ In particular, genetic variants linked to reduced expression of the FADS1 gene have been associated with lower circulating concentrations of EPA. ${ }^{[8,9]}$

Obesity is highly prevalent worldwide and is associated with debilitating and life-threatening comorbidities that can be potentially impacted by $n-3$ PUFAs, 
51 such as cardiovascular disorders. Roux-en-Y gastric bypass (RYGB) is the most

52 effective approach available for the treatment of obesity and its metabolic comorbidities. ${ }^{[10]}$ Aside from limiting food intake and compromising nutrient digestion and absorption, we have shown that the anatomical changes that occur following RYGB may alter the expression of intestinal genes. ${ }^{[11-13]}$

The effect of RYGB on the metabolism of PUFAs by intestinal cells or on n-3 PUFA status remains unclear. We here consider that the restrictive and malabsorptive procedure of RYGB can decrease significantly the systemic availability of $n-3$ PUFAs not only by limiting their ingestion and the intestinal surface for their absorption, but also by impacting on the intestinal expression of genes involved in their endogenous synthesis. Aiming to contribute novel data on this hypothesis, this study assessed the ingestion of PUFAs, intestinal expression of genes involved in PUFA metabolism, and plasma profile of PUFAs before and after RYGB in obese women.

\section{METHODS}

\section{Participants and Ethics}

Twenty adult women (18-60 years old) were screened for eligibility and subsequently admitted for elective RYGB at the Gastrointestinal Surgery Division of the Clinical Hospital at the University of Sao Paulo Medical School (ICHCFMUSP) between April 2010 and March 2014. Inclusion criteria were: BMI $\geq 35$ $\mathrm{kg} / \mathrm{m}^{2}$; proven diagnosis of type 2 diabetes (fasting plasma glucose $\geq 126 \mathrm{mg} / \mathrm{dL}$ and hemoglobin $\mathrm{A} 1 \mathrm{C}>6.5 \%$ ) and/or use of oral antidiabetic medication, and absence of Helicobacter pylori infection. Exclusion criteria were: use of insulin, diagnosis of thyroid or hepatic diseases, subjects undertaking alternative bariatric 
surgery, refusal to participate in the study, current or recent participation in another interventional study.

The study was performed in compliance with the Declaration of Helsinki and Good Clinical Practice guidelines, and was approved by the Ethics Committee of the Hospital das Clínicas of São Paulo Medical (CAPPesq 1011/09). It is registered at www.clinicaltrials.gov (NCT01251016) and Plataforma Brasil (19339913.0.0000.0068). All participants provided written informed consent. This trial is part of the SURMetaGIT (SURgically induced Metabolic effects on the Human Gastrolntestinal Tract) study. ${ }^{[14]}$

\section{Roux-en-Y gastric bypass (RYGB)}

All patients were submitted to RYGB without silicon rings with biliary-pancreatic loops $(50-60 \mathrm{~cm})$ and feed handles $(100-120 \mathrm{~cm})$. RYGB reduces stomach volume by making a proximal gastric pouch roughly $30 \mathrm{~mL}$ in capacity, excluding the rest of the stomach, duodenum, and proximal jejunum from the flow of nutrients. After RYGB, 95\% of ingested food bypasses the stomach, the entire duodenum, and a short portion of the jejunum.

\section{Fat Intake}

Assessments of dietary intake of total PUFAs were performed before RYGB, and three and twelve months after. Food intake was determined via a 7-day dietary record questionnaire $(7 \mathrm{dR})$, including one weekend. The amounts of food were self-recorded by the patient in cooking units (teaspoons, cups, etc.), and analyzed by the software Virtual Nutri Plus. ${ }^{[15]}$ A complimentary food frequency query was 
100

101

102

103

104

105

106

107

108

109

110

111

112

113

114

115

116

117

118

119

120

121

122

123

124

applied to assess the amount and frequency of fish and soybean oil ingestion as the main dietary sources of $n-3$ and n-6 PUFAs, respectively.

\section{Intestinal Biopsies}

Gastrointestinal biopsies (10 to $15 \mathrm{mg}$ of mucosa from stomach, duodenum, jejunum and ileum) were collected by double-balloon enteroscopy (DBE) and immediately immersed in liquid nitrogen before transfer to a $-80^{\circ} \mathrm{C}$ freezer. The biopsy procedure was performed through oral access and under deep sedation preoperatively and again 3 months postoperatively, as described elsewhere. ${ }^{[16]}$ The site of preoperative biopsies was highlighted with India ink SPOT ${ }^{\mathrm{TM}}$ to guide the location for the postoperative collection. All patients had fasted for $12-\mathrm{h}$ and had not used oral drugs (apart from antihypertensive drugs) for 3 to 5 days prior to biopsy.

\section{FADS Gene Expression}

Intestinal biopsies were submitted to RNA extraction using RNasy Plus kit (Qiagen $^{\mathrm{TM}}$ ). Expression of FADS1, FADS2, ELOVL2, and ELOVL5 genes was assessed by using the Human GeneChip 1.0 ST Array (Affymetrix ${ }^{T M}$, Inc., Santa Clara, USA) for each intestinal segment and time-point (pre- and postoperative) in each patient, according to manufacturer's guidelines. Only samples with adequate mRNA ( $\geq 100 \mathrm{ng} / \mathrm{\mu L}$ ) and/or RNA Integrity Number $\geq 7$ (cutoff suggested by the microarray manufacturer), for both pre- and postoperative matching biopsies were considered for microarray analysis. Array quality was checked by boxplot, correlation, and principal component analysis using the R/Bioconductor program (www. bioconductor.org). ${ }^{[17]}$ The significance of 
125 microarrays and rank product methods were analyzed to select differentially expressed genes, using the criterion of $p<0.05$ (corrected for false discovery

127 rate). Expression values were obtained by RMA (Robust multiarray average) preprocessing data. Combat method (http://jlab.byu.edu/ComBat/Abstract.html) was applied for batch effects removal. All tools listed above are available in the R/ Bioconductor program (http://www.bioconductor.org/). Some tissue samples were excluded from the microarray analysis due failure in biopsy collection, in obtaining adequate RNA, or in normalization test. At the end, microarray analysis were applied in samples paired by time-point (pre- and postoperative) of 12 duodenum ( $n=24)$, 16 jejunum $(n=32)$ and 11 ileum $(n=22)$. Significant changes in gene expression were validated by quantitative RT-PCR using the 136 TaqMan gene expression assay (Life Technologies, Carlsbad, CA, USA), according to the manufacturer's recommendations. As RT-PCR requires less restrictive RNA quality and because we included biopsies collected from 5 other obese women that attended the same inclusion criteria applied to those 20 women studied in the RT-qPCR analysis to extend gene validation for an external population, it was applied in samples paired by time-point (pre- and postoperative) of 21 duodenum ( $n=42), 25$ jejunum $(n=50)$ and 23 ileum $(n=$

46). $\beta$-actin was used as the reference gene, based on the results of a pilot study testing this and other genes (18S, glyceraldehyde 3-phosphate dehydrogenase, and beta 2 myoglobin) to identify the most suitable reference for these tissue samples. 


\section{Fatty acid measurements}

151 Plasma $(250 \mu \mathrm{L})$ was obtained from blood samples collected after a 10 -h fast and 152 stored at $-80^{\circ} \mathrm{C}$ until analysis. Collections were performed before RYGB, and 153 three and twelve months after. Plasma total lipids were extracted using 154 chloroform:methanol $(2: 1)$ and phospholipids were isolated by thin layer 155 chromatography using a mixture of hexane: ethyl ether: acetic acid (90: 30: 1), 156 according to the method of Folch et al. ${ }^{[18]}$ Fatty acid methyl esters were prepared 157 by incubation with $140 \mathrm{~g} / \mathrm{L}$ of boron trifluoride in methanol at $80^{\circ} \mathrm{C}$ for $60 \mathrm{~min}$. 158 Subsequent to the extraction process, the fatty acid methyl esters were dried and separated by gas chromatography (Shimadzu Model GC-2010) with flame ionization detection, and an Omegawax 250 (Supelco) column. The operating

conditions of the column corresponded to an initial temperature of $180^{\circ} \mathrm{C}(1 \mathrm{~min})$ and then $270^{\circ} \mathrm{C}(5 \mathrm{~min})$, with a total run time of $36 \mathrm{~min}$. Fatty acid methyl esters were identified and quantified by comparison with external standards. Individual PUFAs (ARA, ALA, EPA and DHA), and ARA/EPA ratio were assessed.

\section{Statistical Analysis}

This study comprises a secondary outcome of a larger clinical trial that aims to analyze changes in the expression of genes encoding Gl hormones related to postoperative glycemic homeostasis. ${ }^{[14]}$ The sample size was calculated using parametric (ANOVA) and non-parametric (Wilcoxon) tests. Effect size was calculated by considering GI hormone variations to be approximately twice in the postoperative versus the preoperative period. Specifically, for gene expression, statistical analysis showed the $\alpha$-error (type I error) of 0.05 and $\beta$-error (type II 
174 error) of 0.2 (power of 0.8 with an effect size $\geq 1.35$ (i.e., 1.35 times the standard

175 deviation of the difference) in a minimum sample size of seven patients. Fatty 176 acid and FFQ data were analyzed by ANOVA using SPSS 18.0 for Windows software (SPSS, Chicago, IL, USA).

\section{RESULTS}

\section{Participant Characteristics}

181 Twenty patients (mean age $46.9 \pm 6.2$ years) were enrolled according to the 182 inclusion criteria and all of them completed the study. Nevertheless, three patients did not present adequate venous access at any time point studied to enable blood collection for plasma fatty acid analysis and one patient did not attend the nutritional consultation after 12 months of RYGB for fat intake recording. Descriptive baseline data of the studied patients are shown in Table 1.

187 Compared to the preoperative period, patients had a significant weight loss and a lower BMI in the postoperative period, with a greater effect at 12 months than 3 months following RYGB.

\section{Fat intake}

192 Table 2 shows data on total PUFAs, fish and soybean oil intakes and the differences observed between the studied time points. The mean PUFA intake was significantly and progressively reduced 3 and 12 months after RYGB, in relation to the preoperative period and between both postoperative periods. The mean fish and soybean oil intakes decreased significantly 3 and 12 months after

197 RYGB, with no difference between the postoperative periods. The number of patients who ingested fish more than once a week decreased after 3 months of 
199 RYGB compared to the preoperative period, with no difference between the

200

201

202

203

204

205

206

207

208

209

210

211

212

213

214

215

216

217

218

219

220

221

222

223 postoperative periods. The number of patients ingesting soybean oil $>1$ time per day decreased significantly after 12 months of RYGB, in comparison to the preoperative period and 3 months postoperatively.

\section{Intestinal biopsies}

The total tissue amount obtained from pre- and postoperative intestinal biopsies was adequate to allow gene expression analysis in all enrolled patients. Success in tissue collection and obtaining adequate mRNA from the different intestinal segments matching pre- and postoperative periods were $70 \%$ for duodenum, $80 \%$ for jejunum, and $60 \%$ for ileum.

\section{Intestinal Gene Expression}

Data on intestinal gene expression are provided in Table 3. Microarray analysis identified significantly decreased expression of FADS1, FADS2 and ELOVL5 three months after RYGB, in comparison to the preoperative period. No significant changes were found for ELOVL2 expression. RT-qPCR analysis comparing pre- and postoperative periods confirmed the changes observed by microarray. However, only the reduction of $F A D S 1$ gene expression in duodenum and jejunum was found to be statistically significant by the RT-qPCR validation $(p<0.050)$.

\section{Fatty acid measurements}

Plasma phospholipid concentrations $(\mu \mathrm{g} / \mathrm{mL})$ of the studied PUFAs are shown in Figure 2 and their individual patterns are shown in Figure 3. ALA and EPA were 
224 significantly lower 3 and 12 months postoperatively compared to preoperatively.

225 EPA was higher 12 months postoperatively compared to 3 months $(p=0.030)$.

226 The ratio of ARA to EPA was lower at preoperative than 3 and 12 months after RYGB (19.6 [8.9-31.8] vs. 40.9 [19.6-65.5] vs. 33.0 [15.1-53.3], respectively; $\mathrm{p}<$ 0.001). Furthermore, this was higher at postoperative 3-month than 12-month period $(p<0.001)$. There were no differences in plasma concentrations of DHA or ARA at any studied period $(p>0.050)$.

Median [1Q;3Q] of preoperative proportion of plasma phospholipid were

$1.11 \%[0.96 ; 1.32]$ DHA. Both ALA and EPA were significantly decreased at 3 months postoperatively $(0.38 \%[0.32 ; 0.45] ; p=0.002$ and $0.22 \%[0.18 ; 0.29] ; p$ $<0.001$, respectively) and EPA but not ALA remained significantly decreased at 12 months postoperatively $(0.47 \%$ [0.40; 0.59$] ; p=0.448$ and $0.26 \%[0.22 ; 0.58]$; $p<0.001$, respectively), in relation to the preoperative period. Comparison between postoperative periods showed that both ALA and EPA were significantly lower at 3 months versus 12 months after RYGB $(p=0.006$ and $p=0.008$, respectively). On the other hand, ARA and DHA were significantly increased at 3 months postoperatively $(10.01 \%$ [8.35;10.94]; $p<0.001$ and $1.38 \%[1.21 ; 1.64]$; $p=0.001$, respectively), in comparison to the preoperative period. However, ARA but not DHA was significantly increased at 12 months postoperatively $(9.17 \%$ [8.13;10.11]; $p=0.006$ and $1.31 \%[1.02 ; 1.68] ; p=0.117)$, in relation to the preoperative period. Comparison between postoperative periods showed that ARA but not DHA was higher at 3 months versus 12 months after RYGB ( $p=$ 0.006 and $p=0.156$, respectively). 


\section{DISCUSSION}

This study identified a significant decrease in the systemic levels of EPA and its precursor ALA following RYGB. This change was accompanied by a longterm decrease in PUFA and fish intake and a decreased intestinal expression of the FADS1 gene 3 months after the surgery. Our findings suggest that multiple factors induced by the anatomical restrictive and malabsorptive changes caused by RYGB may contribute for the systemic depletion of $n-3$ PUFAs. These include decreased intake of both ALA and EPA, likely decreased absorption of both ALA and EPA, and the possibility of decreased biosynthesis in intestinal cells of EPA from ALA. The latter could be due to lower availability of ALA as substrate and also to lower expression of FADS1.

Currently, plasma n-3 PUFA status is not commonly assessed in clinical practice. Although guidelines have not yet established normal serum/plasma ranges, large clinical studies have shown that serum or plasma phospholipid EPA plus DHA among U.S. adults, not taking n-3 PUFA supplements, is about 3\%$4 \%$, although it can vary substantially based on an individual's dietary intake and endogenous metabolism. ${ }^{[19,20]}$ Our patients presented a comparatively very low median of EPA plus DHA (near to 1.5\%) at all the studied periods, highly suggestive of a systemic depletion of these PUFAs even before surgery.

Although high ALA intake has been shown to increase the biosynthesis of EPA for further DHA generation, this conversion is low in humans and most endogenous EPA and DHA are derived from dietary consumption particularly from fatty fish. ${ }^{[21-24]}$ Our patients did not reach the guideline recommendations of EPA and DHA-rich fish intake at any stage of the study. ${ }^{[25]}$ Furthermore, the food intake restriction caused by the RYGB resulted in a significant decrease of fish 
274 consumption as well as in general PUFAs and soybean oil (an important dietary source of both ALA and the ARA precursor LA) ingestion until 12 months postoperatively. Despite several published reports about the effect of RYGB on nutritional deficiencies ${ }^{[11,12]}$, the impact on $\mathrm{n}-3$ PUFA status remains poorly explored. We were able to identify only one previous clinical trial on this topic, which reported changes in the proportion of systemic PUFAs 1 and 6 months following RYGB. ${ }^{[26]}$ Although the authors found no changes in ALA, a persistent decreased EPA and transient increased ARA and DHA proportions along with a low but stable intake of n-3 PUFAs from the preoperative period were observed after the surgical procedure. ${ }^{[26]}$ These findings on proportions of PUFAs are quite similar to ours. We found a persistent decrease and transient increase in EPA and DHA proportions after the surgery, respectively. Furthermore, ALA proportion was decreased only at postoperative 3 months and although ARA proportions were increased from baseline post-surgery, ARA was significantly lower at postoperative 12 months than 3 months.

We consider that our patients' low plasma EPA and DHA levels are in line with their food intake profile, together with the postoperative systemic depletion of ALA and EPA, but this does not fully explain why ARA and DHA levels were not negatively affected by RYGB; indeed, their proportions were even increased after the procedure, at least transiently. One potential explanation for the changes in proportions of n-3 PUFAs (including DHA) could be the decreased expression of the FADS1 gene in parallel to the maintenance of ELOVL2 expression observed at 3 months after RYGB in all the intestinal segments studied, mainly in the duodenum and jejunum. FADS1 gene encodes a rate-limiting enzyme for the conversion of ALA to EPA and changes in its expression strongly affect n-3 
299 PUFA levels in plasma and erythrocytes, regardless of marine fish intake. ${ }^{[27]}$ The enzyme encoded by ELOVL2 catalyzes the reaction that elongates EPA for DHA biosynthesis. According to our data, intestinal conversion of ALA into EPA may be decreased by the loss of expression of the FADS 1 gene, while the conversion of dietary EPA to DHA may be maintained, as the expression of ELOVL2 was unchanged and even slightly but not significantly increased. ${ }^{[28]}$ This also would use EPA, further contributing to its lower plasma concentration.

To our knowledge, this is the first study showing a downregulation of the gene encoding the FADS1 enzyme at multiple intestinal sites following RYGB.

One would expect increased ALA and decreased plasma EPA and DHA levels due to an impaired intestinal conversion of ALA to EPA. Restriction of the amount of PUFAs, soybean oil and fish ingested after RYGB is an inherent bias in our model, since it can contribute to decrease both ALA and EPA levels and make it difficult to confirm the potential for FADS1 participation in this depletion. ${ }^{27}$ However, while changes in dietary intake cannot fully explain all alterations in postoperative PUFA levels, decreased intestinal expression of the FADS1 gene is in line with the plasma proportion of the studied n-3 PUFAs observed after RYGB. Regarding the systemic ARA response to RYGB, the enzyme encoded by the FADS1 gene also participates in the biosynthesis of this PUFA and variations in its cluster are associated with the systemic ARA depletion. ${ }^{[29]}$ In common with these findings, instead of observing a concomitant decrease of intestinal expression of FADS1 and systemic ARA levels, the plasma proportion of this PUFA was increased in our patients 3 months after RYGB, compared to the preoperative period and 12 months postoperatively. A transiently increased proportion of ARA after RYGB observed in our study and in other trial may be as 
324 a result of the surgery causing weight loss, with high triglyceride breakdown. ${ }^{[26]}$

325 Stored triglycerides contain a fairly high proportion of n-6 PUFAs, especially LA, 326 the precursor of ARA, but also ARA. ${ }^{[30]}$ Furthermore, in obese patients, stored 327 triglycerides may be particularly rich in $n-6$ PUFAs, since higher dietary $n-6: n-3$ PUFA ratios are associated with obesity. ${ }^{[31]}$ As a result, increased plasma ARA proportion might occur with weight loss, mainly in the early postoperative period where weight loss was more pronounced in our patients. A recent study reported a ratio of n-6:n-3 PUFAs close to 5:1, with a higher mean amount $(\mathrm{mg} / \mathrm{g})$ of LA and ARA than ALA and EPA (72.8 and 4.7 vs. 8.0 and 1.2 , respectively), in the subcutaneous adipose tissue of obese patients before undertaking RYGB. ${ }^{[32]}$ Although the authors also had assessed changes in the systemic levels of phospholipid PUFAs after 12 months of surgery, comparison with our data is difficult because the studied individuals were instructed to consume rapeseed oil ( 3 tea spoons and 6 tea spoons of mainly rapeseed oil based spreads daily) and fish (2-3 times a week) during all the assessed postoperative periods. ${ }^{[32]}$ They observed an increase of ALA (one of the major PUFAs in rapeseed oil), no changes in EPA and a decrease of ARA and DHA proportions in serum phospholipids in response to RYGB, and suggested that postoperative dietary intervention influenced their findings. ${ }^{[32]}$

Severe obesity is accompanied by a chronic low-grade inflammation and disturbances in lipid metabolism linked with most of its comorbidities, such as type 2 diabetes mellitus, hypertension and dyslipidemia. ${ }^{[33]}$ It is widely recognized that $n-3$ and $n-6$ PUFAs can distinctly impact on inflammation and lipid metabolism by mechanisms that include modulation of gene transcription. ${ }^{[34]}$ 
348 Particularly, ARA (n-6 PUFA) has pro-inflammatory and detrimental lipogenic properties, while the EPA and DHA (n-3 PUFAs) have opposite effects. ${ }^{[34]}$ In accordance with their biological properties, high n-6 fatty acid levels are associated with an increased risk of obesity, whereas a high concentration of n3 fatty acids in phospholipids is associated with reduced risk. ${ }^{[35]}$ The postoperative changes in levels of PUFAs observed in this study resulted in an increased ARA:EPA ratio seen until 12 months postoperatively, in comparison to the preoperative period. As high ARA:EPA ratios are shown to be harmful for human health, our data are suggestive that by decreasing n-3 PUFAs, RYGB might create an unfavorable systemic PUFA profile. ${ }^{[36-38]}$

RYGB has shown metabolic effects that counteract some obesityassociated comorbidities. ${ }^{[39]}$ In particular, obese patients with type 2 diabetes can experience glycemic homeostasis early after the procedure, but not all of them achieve the postoperative remission of the disease and some can experience its relapse longer term. ${ }^{[40-43]}$ It is possible that the unfavorable PUFA profile induced by RYGB may impair the achievement and maintenance of the metabolic benefits expected to be induced surgically.

It has been consistently demonstrated that higher plasma levels of EPA and DHA are crucial in maintaining positive health outcomes by preventing and treating metabolic and inflammatory disorders. ${ }^{[3,44-46]}$ Large observational studies have been homogenous in terms of these findings, with systematic reviews and meta-analyses showing that higher consumption of fish and higher dietary or plasma levels of n-3 PUFAs are associated with a lower risk of chronic disorders, in particular of cardiovascular diseases. ${ }^{[47-51]}$ All of these benefits may aid the 
372 recovery of obese patients undergoing RYGB and favor the postoperative

373 resolution of their obesity-related comorbidities. ${ }^{[52]}$

We propose that n-3 PUFA supplementation may be required following RYGB to counteract the surgery induced reduction in n-3 PUFA status. Notably, according to our data, systemic n-3 PUFA depletion seems to occur before RYGB, and therefore patients may benefit from supplementation before the procedure. In a double blind randomized trial, the supplementation of n-3 PUFAs over a period of 8 weeks before bariatric surgery was associated with improvements in circulating and adipose tissue (visceral and subcutaneous) markers of inflammation and in lipid metabolism in severely obese nondiabetic patients. ${ }^{[53]}$ In another study, 4-weeks of preoperative supplementation with n-3 PUFAs reduced the liver volume of severely obese patients and facilitated the access to the gastro-esophageal junction during laparoscopic RYGB surgery by promoting an easy retraction of the left hepatic lobe. ${ }^{[54]}$

Our study has some limitations. Although the seven-day nutritional record showed a significant reduction in total PUFAs and fish intake in both postoperative periods after RYGB, the individual intake of n-3 PUFAs (ALA, EPA, DHA) was not measured. Also, the intestinal expression of fatty acid desaturase and elongase genes was not performed at 12 months postoperatively and was not validated at the protein level. Furthermore, the investigation of individual genetic variants of $F A D S 1$ gene would be necessary to clarify the repercussion of our findings on the endogenous n-3 fatty acid metabolism. intake, anatomical changes following RYGB might decrease the intestinal ability to synthesize bioactive $\mathrm{n}-3$ fatty acids through reduced $F A D S 1$ gene expression. 
397 Although it is uncertain if these alterations work together to cause the 398 postoperative systemic depletion of ALA and EPA, the supplementation of n-3 399 PUFAs might be considered for obese patients who are candidates for RYGB to 400 avoid the risk of $n-3$ deficiency and potentially improve their postoperative 401 recovery.

402

403

CONFLICTS OF INTEREST

404 The authors have declared that no competing interests exist.

\section{FUNDING}

406 This study was supported by Fundação de Amparo à Pesquisa do Estado de São 407 Paulo (FAPESP) project (no. 2011/09612-3)

\section{REFERENCES}

1. Martinelli N, Girelli D, Malerba G, Guarini P, Illig T, Trabetti E, Sandri M, Friso S, Pizzolo F, Schaeffer L, et al. FADS genotypes and desaturase activity estimated by the ratio of arachidonic acid to linoleic acid are associated with inflammation and coronary artery disease. Am J Clin Nutr. 2008; 88:941-9.

2. Baker EJ, Miles EA, Burdge GC, Yaqoob P, Calder PC. Metabolism and functional effects of plant-derived omega-3 fatty acids in humans. Prog Lipid Res. 2016; 64:30-56.

3. Calder PC, Deckelbaum RJ. Dietary lipids: more than just a source of calories. Curr Opin Clin Nutr Metab Care.1999; 2:105-7. 
4. Simopoulos AP. Genetic variants in the metabolism of $n-6$ and omega-3 fatty acids: their role in the determination of nutritional requirements and chronic disease risk. Exp Biol Med. 2010, 235:785-95.

5. Roke K, Walton K, Klingel SL, Harnett A, Subedi S, Haines J, Mutch DM. Evaluating Changes in Omega-3 Fatty Acid Intake after Receiving Personal FADS1 Genetic Information: A Randomized Nutrigenetic Intervention. Nutrients. 2017; 9: 240.

6. Beguin P, Schneider AC, Mignolet E, Schneider YJ, Larondelle Y. Polyunsaturated fatty acid metabolism in enterocyte models: T84 cell line vs. Caco-2 cell line. In Vitro Cell Dev Biol Anim. 2014; 50(2), 111-120.

7. Gillingham LG, Harding SV, Rideout TC, Yurkova N, Cunnane SC, Eck PK, Jones PJ. Dietary oils and FADS1-FADS2 genetic variants modulate [13C] a-linolenic acid metabolism and plasma fatty acid composition. Am J Clin Nutr. 2013; 97:195-207.

8. Tanaka T, Shen J, Abecasis GR, Kisialiou A, Ordovas JM, Guralnik JM, Singleton A, Bandinelli S, Cherubini A, Arnett D, et al. Genome-wide association study of plasma polyunsaturated fatty acids in the inchianti study. PLoS Genet. 2009; 5: e1000338.

9. Heimerl S, Moehle C, Zahn A, Boettcher A, Stremmel W, Langmann T, Schmitz G. Alterations in intestinal fatty acid metabolism in inflammatory bowel disease. Biochim Biophys Acta. 2006; 1762:341-50.

10. Cavin JB, Couvelard A, Lebtahi R, Ducroc R, Arapis K, Voitellier E, Cluzeaud F, Gillard L, Hourseau M, Mikail N, et al. Differences in Alimentary Glucose Absorption and Intestinal Disposal of Blood Glucose 
After Roux-en-Y Gastric Bypass vs Sleeve Gastrectomy. Gastroenterology. 2016; 150:454-64.

11. Dogan K, Homan J, Aarts EO, de Boer H, van Laarhoven CJ, Berends FJ. Long-term nutritional status in patients following Roux-en-Y gastric bypass surgery. Clin Nutr. 2017; 17: 0261-5614.

12. Sala P, Torrinhas RS, Fonseca DC, Heymsfield S, Giannella-Neto D, Waitzberg DL. Type 2 Diabetes Remission After Roux-en-Y Gastric Bypass: Evidence for Increased Expression of Jejunal Genes Encoding Regenerating Pancreatic Islet-Derived Proteins as a Potential Mechanism. Obes Surg. 2017; 27:1123-7.

13. Sala P, Belarmino G, Torrinhas RS, Machado NM, Fonseca DC, Ravacci GR, Ishida RK, Guarda IF, de Moura EG, Sakai P, et al. Gastrointestinal Transcriptomic Response of Metabolic Vitamin B12 Pathways in Roux-enY Gastric Bypass. Clin Transl Gastroenterol. 2017; 8:e212.

14. Sala P, Belarmino G, Machado NM, Cardinelli CS, Al Assal K, Silva MM, Fonseca DC, Ishida RK, Santo MA, de Moura EG, et al. The SURMetaGIT study: design and rationale for a prospective pan-omics examination of the gastrointestinal response to Roux-en-Y gastric bypass surgery. $J$ Int Med Res. 2016; 44:1359-75.

15. Virtual Nutri Plus®. Software de Nutrição. Disponível em http://www.virtualnutriplus.com.br/

16. Kuga R, Safatle-Ribeiro AV, Faintuch J, Ishida RK, Furuya CK Jr, Garrido AB Jr, Cecconello I, Ishioka S, Sakai P. Endoscopic Findings in the Excluded Stomach After Roux-en-Y Gastric Bypass Surgery. Arch Surg. $2007 ; 142: 942-6$. 
17. Manual da Affymetriz. Gene Chip 1.0 ST Human. Disponível em: http://media.affymetrix.com/support/technical/datasheets/gene_1_0_st _datasheet.pdf.

18. Folch J, Lees, M. \& Stanley, G.H.S. A simple method for the isolation and purification of total lipids from animal tissues. J Biol Chem. 1957; 226:497509.

19. Brasky TM, Till C, White E, Neuhouser ML, Song X, Goodman P, Thompson IM, King IB, Albanes D, Kristal AR. Serum phospholipid fatty acids and prostate cancer risk: results from the prostate cancer prevention trial. Am J Epidemiol. 2011; 173:1429-39.

20. Brasky TM, Darke AK, Song X, Tangen CM, Goodman PJ, Thompson IM, Meyskens FL Jr, Goodman GE, Minasian LM, Parnes HL, et al. Plasma phospholipid fatty acids and prostate cancer risk in the SELECT trial. $J$ Natl Cancer Inst. 2013; 105:1132-41.

21. Hussein N, Ah-Sing E, Wilkinson P, Leach C, Griffin BA, Millward DJ. Long-chain conversion of [13C]linoleic acid and alpha-linolenic acid in response to marked changes in their dietary intake in men. $J$ Lipid Res. 2005; 46:269-80.

22. Burdge G. Alpha-linolenic acid metabolism in men and women: nutritional and biological implications. Curr Opin Clin Nutr Metab Care. 2004; 7(2):137-44.

23. Arterburn LM, Hall EB, Oken H. Distribution, interconversion, and dose response of $\mathrm{n}-3$ fatty acids in humans. Am J Clin Nutr. 2006; 83:1467S$76 S$. 
24. Plourde M, Cunnane SC. Extremely limited synthesis of long chain polyunsaturates in adults: implications for their dietary essentiality and use as supplements. Appl Physiol Nutr Metab. 2007;32:619-34.

25. Global Organization for EPA and DHA Omega-3. Global Recommendations for EPA and DHA Intake; Global Organization for EPA and DHA Omega-3: Salt Lake City, UT, USA, 2014.

26. Forbes R, Gasevic D, Watson EM, Ziegler TR, Lin E, Burgess JR, GletsuMiller N. Essential Fatty Acid Plasma Profiles Following Gastric Bypass and Adjusted Gastric Banding Bariatric Surgeries. Obes Surg. 2016; $26: 1237-46$

27. Takkunen MJ, de Mello VD, Schwab US, Kuusisto J, Vaittinen M, Ågren JJ, Laakso M, Pihlajamäki J, Uusitupa MI. Gene-diet interaction of a common FADS1 variant with marine polyunsaturated fatty acids for fatty acid composition in plasma and erythrocytes among men. Mol Nutr Food Res. 2016; 60:381-9.

28. Alsaleh A1, Maniou Z, Lewis FJ, Hall WL, Sanders TA, O'Dell SD. ELOVL2 gene polymorphisms are associated with increases in plasma eicosapentaenoic and docosahexaenoic acid proportions after fish oil supplement. Genes Nutr. 2014; 9:362.

29. Roke K, Ralston JC, Abdelmagid S, Nielsen DE, Badawi A, El-Sohemy A, Ma DW, Mutch DM. Variation in the FADS1/2 gene cluster alters plasma n-6 PUFA and is weakly associated with hsCRP levels in healthy young adults. Prostaglandins Leukot Essent Fatty Acids. 2013; 89:257-63. 
30. Calder PC, Harvey DJ, Pond CM, Newsholme EA. Site-specific differences in the fatty acid composition of human adipose tissue. Lipids. 1992; 27(9):716-20.

31. Simopoulos AP. An Increase in the N-6/Omega-3 Fatty Acid Ratio Increases the Risk for Obesity. Nutrients. 2016; 8:128.

32. Walle $P$, Takkunen $M$, Männistö V, Vaittinen M, Käkelä $P$, Ågren J, Schwab U, Lindström J, Tuomilehto J, Uusitupa M, et al. Alterations in fatty acid metabolism in response to obesity surgery combined with dietary counseling. Nutr Diabetes. 2017;7:e285.

33. Monteiro R, Azevedo I. Chronic Inflammation in Obesity and the Metabolic Syndrome. Mediators Inflamm. 2010; 2010:289645.

34. Schmitz G, Ecker J. The opposing effects of $n-3$ and n-6 fatty acids. Prog Lipid Res. 2008; 47:147-55.

35. Wang L, Manson JE, Rautiainen S, Gaziano JM, Buring JE, Tsai MY, Sesso HD. A prospective study of erythrocyte polyunsaturated fatty acid, weight gain, and risk of becoming overweight or obese in middle-aged and older women. Eur J Nutr. 2016; 55:687-97.

36. Ishitobi T, Hyogo H, Kan H, Hiramatsu A, Arihiro K, Aikata H, Chayama K. Eicosapentaenoic acid/arachidonic acid ratio as a possible link between non-alcoholic fatty liver disease and cardiovascular disease. Hepatol Res. $2015 ; 45: 533-9$.

37. Shoji T, Kakiya R, Hayashi T, Tsujimoto Y, Sonoda M, Shima H, Mori K, Fukumoto S, Tahara $H$, Shioi A, et al. Serum n-3 and n-6 polyunsaturated fatty acid profile as an independent predictor of cardiovascular events in hemodialysis patients. Am J Kidney Dis. 2013; 62:568-76. 
38. Inoue K, Kishida K, Hirata A, Funahashi T, Shimomura I. Low serum eicosapentaenoic acid / arachidonic acid ratio in male subjects with visceral obesity. Nutr Metab (Lond). 2013; 10:25.

39. Ramos-Leví AM, Rubio Herrera MA. Metabolic surgery: quo vadis? Endocrinol Nutr. 2014; 61:35-46.

40. Dixon JB, le Roux CW, Rubino F, Zimmet P. Bariatric surgery for type 2 diabetes. Lancet. 2012; 379:2300-11.

41. Schauer PR, Burguera B, Ikramuddin S. Effect of laparoscopic Roux-en Y gastric bypass on type 2 diabetes mellitus. Ann Surg. 2003; 238:467-84.

42. Buchwald H, Avidor Y, Braunwald E, Jensen MD, Pories W, Fahrbach K, Schoelles K. Bariatric surgery: a systematic review and meta-analysis. JAMA. 2004; 292:1724-37.

43. Arterburn DE, Bogart A, Sherwood NE, Sidney S, Coleman KJ, Haneuse S, O'Connor PJ, Theis MK, Campos GM, McCulloch D, et al. A multisite study of long-term remission and relapse of type 2 diabetes mellitus following gastric bypass. Obes Surg. 2013; 23:93-102.

44. Itariu BK, Zeyda M, Leitner L, Marculescu R, Stulnig TM. Treatment with n-3 polyunsaturated fatty acids overcomes the inverse association of vitamin D deficiency with inflammation in severely obese patients: A Randomized Controlled Trial. PLOS ONE. 2015; 8:54634.

45. de Castro GS, Calder PC. Non-alcoholic fatty liver disease and its treatment with n-3 polyunsaturated fatty acids. Clin Nutr. 2017; 17:0261561430011-0. 
46. Feskens $\mathrm{EJ}$, Bowles $\mathrm{CH}$ and Kromhout D. Inverse association between fish intake and risk of glucose intolerance in normoglycemic elderly men and women. Diabetes Care. 1991; 14:935-41.

47. Djousse L, Akinkuolie AO, Wu JH, Ding EL, Gaziano JM. Fish consumption, omega-3 fatty acids and risk of heart failure: a metaanalysis. Clin Nutr. 2012; 31:846-53.

48. Del Gobbo LC, Imamura F, Aslibekyan S, Marklund M, Virtanen JK, Wennberg M, Yakoob MY, Chiuve SE, Dela Cruz L, Frazier-Wood AC, et al. Cohorts for Heart and Aging Research in Genomic Epidemiology (CHARGE) Fatty Acids and Outcomes Research Consortium (FORCe). Omega-3 polyunsaturated fatty acid biomarkers and coronary heart disease: pooling project of 19 cohort studies. JAMA Intern Med. 2016; 176:1155-66.

49. Wang C, Harris WS, Chung M, Lichtenstein AH, Balk EM, Kupelnick B, Jordan HS, Lau J. n-3 Fatty acids from fish or fish-oil supplements, but not alpha-linolenic acid, benefit cardiovascular disease outcomes in primaryand secondary-prevention studies: a systematic review. Am J Clin Nutr. $2006 ; 84: 5-17$

50. Morris MC, Sacks F, Rosner B. Does fish oil lower blood pressure? A meta-analysis of controlled trials. Circulation. 1993; 88:523-33.

51.Tanaka K, Ishikawa $\mathrm{Y}$, Yokoyama M, Origasa H, Matsuzaki M, Saito $\mathrm{Y}$, Matsuzawa Y, Sasaki J, Oikawa S, Hishida H, et al. Reduction in the recurrence of stroke by eicosapentaenoic acid for hypercholesterolemic patients: subanalysis of the JELIS trial. Stroke. 2008; 39:2052-8. 
52. Wang Y, Huang F. N-3 Polyunsaturated Fatty Acids and Inflammation in Obesity: Local Effect and Systemic Benefit. Biomed Res Int. 2015; 2015:581469.

53. Itariu BK, Zeyda M, Hochbrugger EE, Neuhofer A, Prager G, Schindler K, Bohdjalian A, Mascher D, Vangala S, Schranz M, et al. Long-chain n-3 PUFAs reduce adipose tissue and systemic inflammation in severely obese nondiabetic patients: a randomized controlled trial. Am J Clin Nutr. 2012; 96:1137-49.

54. Iannelli A, Martini F, Schneck AS, Ghavami B, Baudin G, Anty R, Gugenheim J. Preoperative 4-week supplementation with omega-3 polyunsaturated fatty acids reduces liver volume and facilitates bariatric surgery in morbidly obese patients. Obes Surg. 2013; 23:1761-5. 
Table 1. Descriptive data of obese women $(n=20)$ before, and three and twelve months following Roux En-Y Gastric Bypass

\begin{tabular}{|c|c|c|c|c|c|c|}
\hline Variable & T0 & $\mathrm{T} 1$ & $\mathrm{~T} 2$ & $P$ value* & $P$ value $e^{¥}$ & $P$ value ${ }^{\phi}$ \\
\hline Weight (kg) & $\begin{array}{c}119.4 \\
(83.5-143.6)\end{array}$ & $\begin{array}{c}95.6 \\
(68.2-114.0)\end{array}$ & $\begin{array}{c}77.3 \\
(63.3-100.5)\end{array}$ & $<0.001$ & $<0.001$ & 0.001 \\
\hline BMI $\left(\mathrm{kg} / \mathrm{m}^{2}\right)$ & $\begin{array}{c}46.4 \\
(37.1-57.5)\end{array}$ & $\begin{array}{c}38.5 \\
(30.3-45.5)\end{array}$ & $\begin{array}{c}32.4 \\
(27.5-40.2)\end{array}$ & $<0.001$ & $<0.001$ & $<0.001$ \\
\hline $\mathrm{FBG}(\mathrm{mg} / \mathrm{dl})$ & $\begin{array}{c}208 \\
(77-338)\end{array}$ & $\begin{array}{c}100 \\
(75-159)\end{array}$ & $\begin{array}{c}90 \\
(68-116)\end{array}$ & $<0.001$ & $<0.001$ & 0.049 \\
\hline $\mathrm{HbA1c}(\%)$ & $9(6-13)$ & $6(5-7)$ & $6(5-7)$ & $<0.001$ & $<0.001$ & 0.019 \\
\hline EWL (\%) \# & 0 & 19.9 & 35.3 & $<0.001$ & $<0.001$ & 0.001 \\
\hline
\end{tabular}

Data are expressed as median (minimum-maximum). T0, preoperative; T1, postoperative 3-month; T2, postoperative 12-month; *T0 vs. T1; ${ }^{¥} \mathrm{~T} 0$ vs. T2; ${ }^{\phi}$ T1 vs. T2. \#EWL, Excess Weight Loss in comparation to T0. BMI, Body Mass Index; FBG, Fasting blood glucose; HbA1c, Hemoglobin A1c. 
Table 2. Dietary intake of total polyunsaturated fatty acids (PUFAs), fish and soybean oil (SO) by obese women before and 3 and 12 months after Roux en-y gastric bypass

\begin{tabular}{|c|c|c|c|c|c|}
\hline & PUFAs (g/day) & Fish (g/week) & SO (mL/day) & $\begin{array}{c}\text { Fish intake } \\
\geq 1 \text { time per week } \\
(\%)\end{array}$ & $\begin{array}{c}\text { SO intake } \\
\geq 1 \text { time per day }(\%)\end{array}$ \\
\hline TO & $12.54 \pm 5.46$ & $133.53 \pm 48.47$ & $13.24 \pm 10.89$ & $45 \%$ & $41 \%$ \\
\hline $\mathrm{T} 1$ & $8.05 \pm 3.15$ & $76.67 \pm 25.82$ & $6.32 \pm 3.96$ & $25 \%$ & $47 \%$ \\
\hline $\mathrm{T} 2$ & $6.23 \pm 1.40$ & $88.12 \pm 36.92$ & $5.00 \pm 4.38$ & $21 \%$ & $12 \%$ \\
\hline$P$ value* & 0.002 & 0.000 & 0.022 & 0.013 & 0.404 \\
\hline$P$ value ${ }^{¥}$ & 0.000 & 0.000 & 0.007 & 0.058 & 0.033 \\
\hline
\end{tabular}

Data expressed as mean \pm standard deviation. T0, preoperative; T1, postoperative 3-month; T2, postoperative 12-month; *T0 vs. T1;

¥ T0 vs. T2; ${ }^{\dagger} \mathrm{T} 1$ vs. T2. 
Table 3: Changes in the intestinal expression of genes encoding key enzymes of the pathway of endogenous biosynthesis of polyunsaturated fatty acids in obese patients after 3 months of Roux en-Y gastric bypass

\begin{tabular}{|c|c|c|c|c|c|c|}
\hline & Duodenum & & Jejunum & & Ileum & \\
\hline Gene & Microarray & $\begin{array}{l}\text { RT-PCR } \\
\text { validation }\end{array}$ & Microarray & $\begin{array}{l}\text { RT-PCR } \\
\text { validation }\end{array}$ & $\begin{array}{l}\text { Microarra } \\
\text { y }\end{array}$ & $\begin{array}{l}\text { RT- } \\
\text { PCR } \\
\text { validati } \\
\text { on }\end{array}$ \\
\hline FADS1 & -0.557 & -1.620 & -0.250 & -1.549 & -0.358 & 1.087 \\
\hline FADS2 & -0.397 & -0.942 & -0.136 & - & -0.187 & - \\
\hline ELOVL2 & +0.016 & - & +0.029 & - & +0.055 & - \\
\hline ELOVL5 & -0.573 & -1.145 & +0.024 & - & -0.166 & - \\
\hline
\end{tabular}

Data correspond to comparison of gene expression between preoperative and 3 months post-operative. Values are fold changes. Negative and positive fold changes indicate decreased and increased expression, respectively. Bold values correspond to significant changes $(P \leq 0.05)$. 
408 Figure 1: Desaturation and elongation of omega-3 and n-6 polyunsaturated fatty acids ${ }^{6}$

409

410

411

\section{Eicosanoids}

41i Mainly proinflammatory

41! prostaglandins, thromboxanes, and

421 leukotrienes and pro-resolving lipoxins) Essential Fatty Acids (EFA)

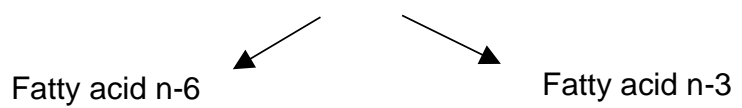
$18: 2 n-6$
$18: 3 n-3$

ELOVL5 Linoleic acid (LA)

$20: 2 n-6$

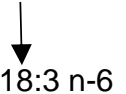

a-Linolenic acid (ALA)

\section{ELOVL5}

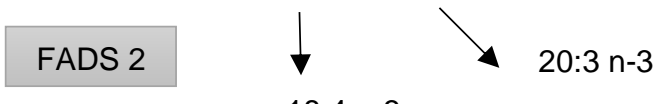

18:4 n-3 Eicosatrienoic acid (ERA)

Stearidonic acid (SDA)

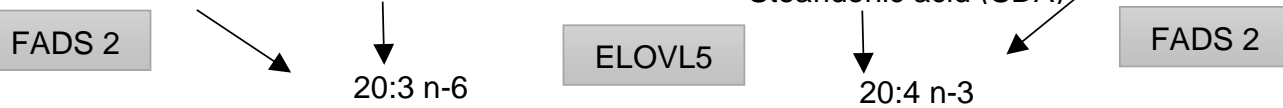

Dihomo-v-Linoleic acid (DGLA) Eicosatetraenoic acid (ETA)

\section{Eicosanoids}

Less inflammatory prostaglandins, thromboxanes and leukotrienes and pro-resolving resolvins

\section{Docosatetraenoic acid (DTA)}

Docosapentaenoic acid (DPA)

$$
\left.\right|_{24: 5 n-3} \text { ELOVL2 }
$$

Tetracosapentaenoic acid (TPA)

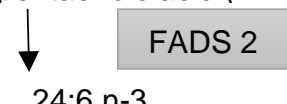

Tetracosahexaenoic acid (THA)

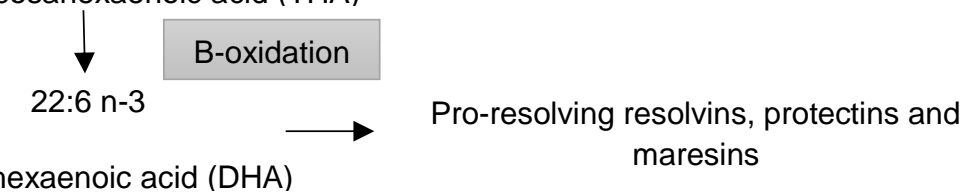


Figure 2: Concentration of fatty acids $(\mu \mathrm{g} / \mathrm{ml})$ in plasma phospholipids in obese women before, 3 and 12 months after Roux en-y gastric bypass

426

427

428

429

430

431

432

433

434

435
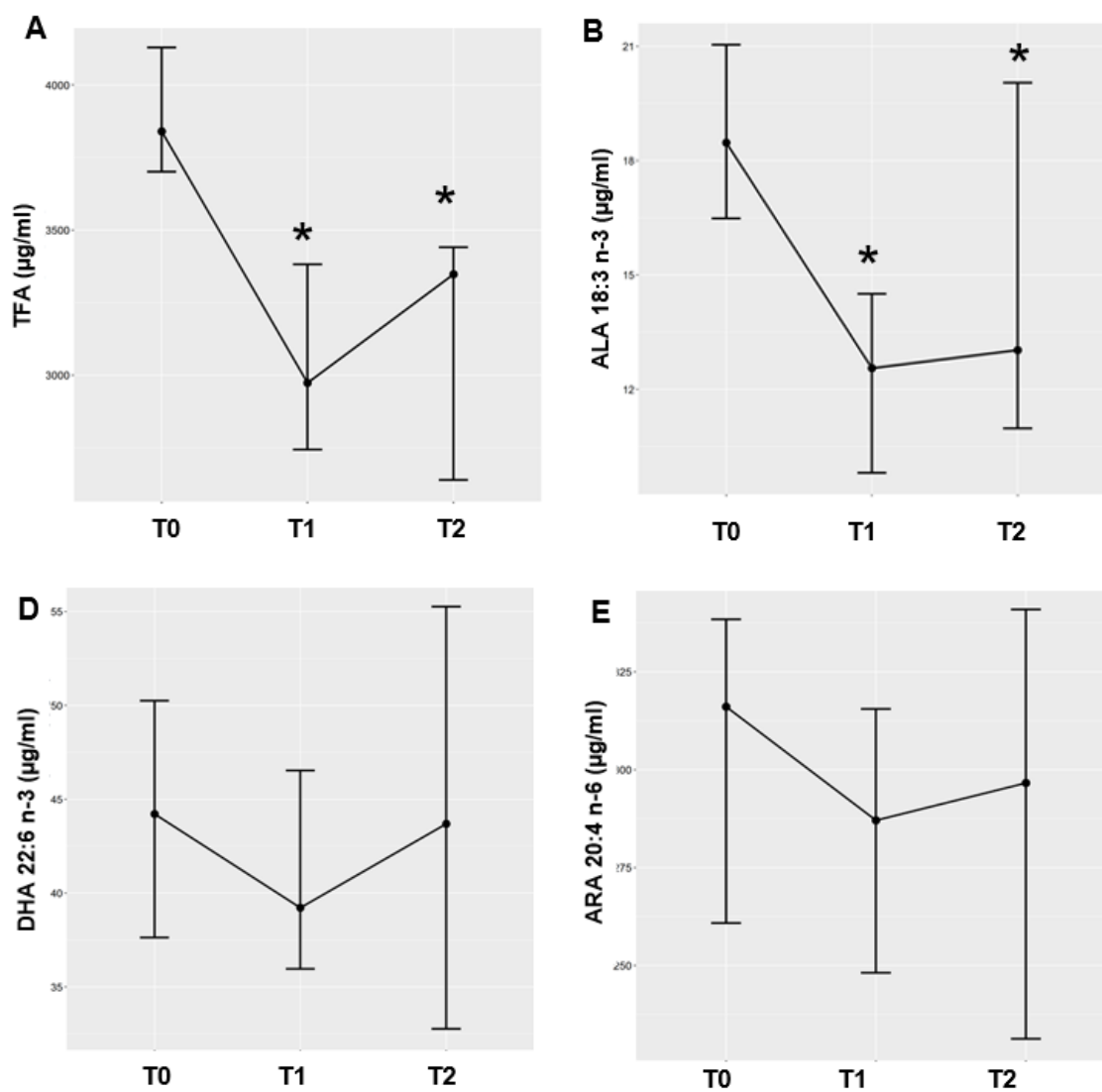

E

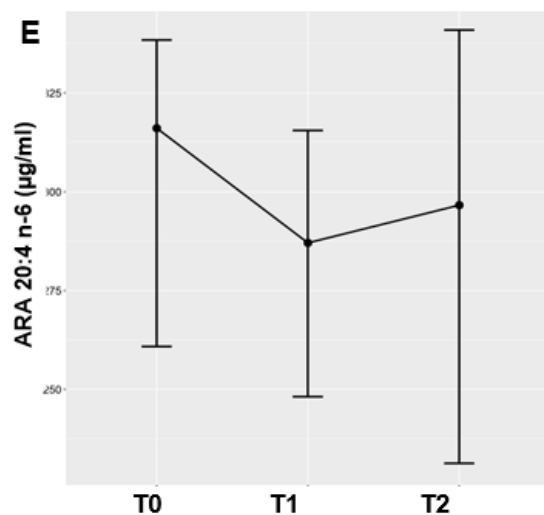

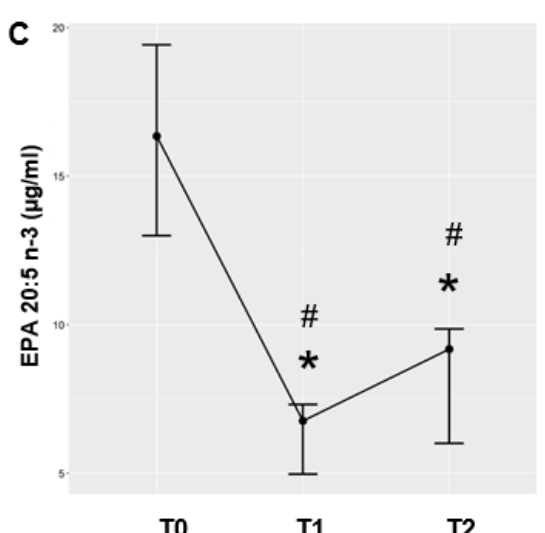

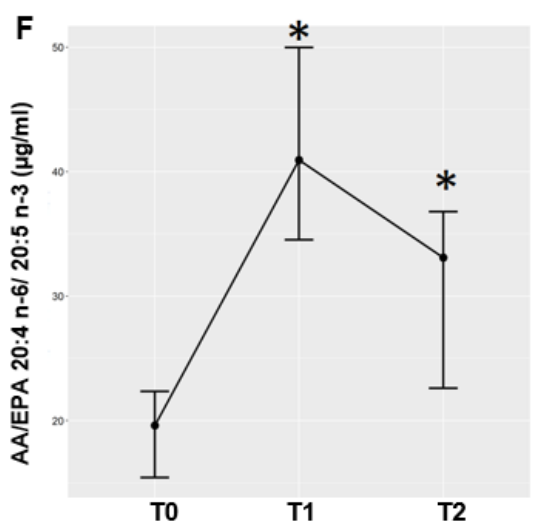


T0, preoperative; T1, postoperative 3-month; T2, postoperative 12-month. The symbol "*” indicates different mean values between postoperative time versus pre-operative. The symbol "\#” indicates different mean values comparing only postoperative periods (T1;T2). A:TFA: total fatty acids; $\left({ }^{*} T 1 ; p<0.001\right) ;\left({ }^{*} T 2 ; p=0.002\right) ; B:$ ALA $(18: 3 n-3)$ : alpha linolenic acid; $\left({ }^{*} T 1 ; p<0.001\right) ;\left({ }^{*} T 2 ; p<\right.$ 0.001); C: EPA (20:5 n-3): eicosapentaenoic acid; ( $\left.{ }^{*} T 1 ; p<0.001\right) ;\left({ }^{*} T 2 ; p<0.001\right) ;(\# T 2 ; p=0.03) ; D:$ DHA (22: 6 n-3): docosahexaenoic acid; E: ARA (20:4 n-6): arachidonic acid; F: ratio ARA/EPA ( $\left.{ }^{*} T 1 ; p<0.001\right) ;\left({ }^{*} T 2 ; p<0.001\right)$. 42 
Figure 3: Individual patterns of changes in plasma phospholipid concentrations $(\mu \mathrm{g} / \mathrm{mL})$ of polyunsaturated fatty acids in obese women before, 3 and 12 months after Roux en-y gastric bypass

450

451

452

453

454

455

456

457

458

459

460

461

462
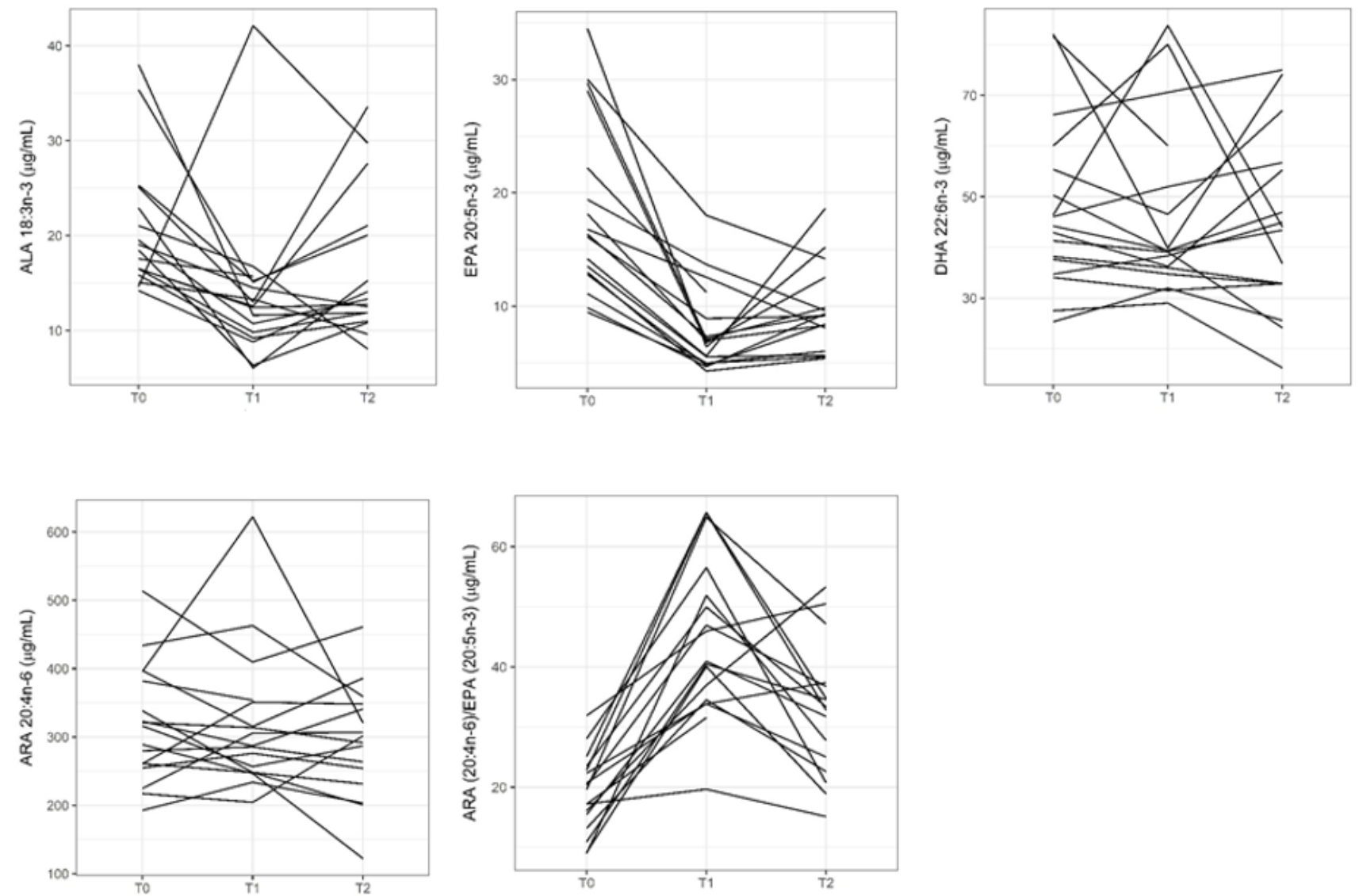
463 T0, preoperative; T1, postoperative 3-month; T2, postoperative 12-month. 